\title{
Phenotypical and Myopathological Consequences of Compound Heterozygous Missense and Nonsense Variants in SLC18A3
}

\author{
Adela Della Marina ${ }^{1, *(1)}$, Annabelle Arlt ${ }^{2}$, Ulrike Schara-Schmidt ${ }^{1}$, Christel Depienne ${ }^{3}$, Andrea Gangfuß ${ }^{1}$, \\ Heike Kölbel ${ }^{1}$, Albert Sickmann ${ }^{4}$, Erik Freier ${ }^{4}$, Nicolai Kohlschmidt ${ }^{2}$, Andreas Hentschel ${ }^{4}$, Joachim Weis ${ }^{5}$, \\ Artur Czech ${ }^{4}$, Anika Grüneboom ${ }^{4,+}+$ and Andreas Roos ${ }^{1,6,+}$
}

check for updates

Citation: Della Marina, A.; Arlt, A.; Schara-Schmidt, U.; Depienne, C.; Gangfuß, A.; Kölbel, H.; Sickmann, A.; Freier, E.; Kohlschmidt, N.; Hentschel, A.; et al. Phenotypical and Myopathological Consequences of Compound Heterozygous Missense and Nonsense Variants in SLC18A3. Cells 2021, 10, 3481. https:/ / doi.org/10.3390/cells10123481

Academic Editors: Anne Schänzer, Tobias Ruck, Andreas Hahn and Corinna Preusse

Received: 17 October 2021 Accepted: 12 November 2021 Published: 9 December 2021

Publisher's Note: MDPI stays neutral with regard to jurisdictional claims in published maps and institutional affiliations.

Copyright: (c) 2021 by the authors. Licensee MDPI, Basel, Switzerland. This article is an open access article distributed under the terms and conditions of the Creative Commons Attribution (CC BY) license (https:/ / creativecommons.org/licenses/by/ $4.0 /)$.
1 Department of Pediatric Neurology, Centre for Neuromuscular Disorders, Centre for Translational Neuro- and Behavioral Sciences, University Duisburg-Essen, 45122 Essen, Germany; Ulrike.Schara-Schmidt@uk-essen.de (U.S.-S.); andrea.gangfuss@uk-essen.de (A.G.);

Heike.Koelbel@uk-essen.de (H.K.); Andreas.Roos@uk-essen.de (A.R.)

2 Institute of Clinical Genetics and Tumor Genetics Bonn, 53111 Bonn, Germany; Annabelle.Arlt@ukmuenster.de (A.A.); kohlschmidt@genetik-bonn.de (N.K.)

3 Institute of Human Genetics, University Hospital Essen, University of Duisburg-Essen, 45122 Essen, Germany; christel.depienne@uk-essen.de

4 Leibniz-Institut für Analytische Wissenschaften -ISAS- e.V., 44139 Dortmund, Germany; albert.sickmann@isas.de (A.S.); erik.freier@ruhr-uni-bochum.de (E.F.); andreas.hentschel@isas.de (A.H.); artur.czech@isas.de (A.C.); anika.grueneboom@isas.de (A.G.)

5 Institute of Neuropathology, University Hospital Aachen, RWTH-Aachen University, 52074 Aachen, Germany; JWeis@ukaachen.de

6 Children's Hospital of Eastern Ontario Research Institute, Ottawa, ON K1H 8L1, Canada

* Correspondence: adela.dellamarina@uk-essen.de

+ The authors contribute equally to this work.

Abstract: Background: Presynaptic forms of congenital myasthenic syndromes (CMS) due to pathogenic variants in SLC18A3 impairing the synthesis and recycling of acetylcholine (ACh) have recently been described. SLC18A3 encodes the vesicular ACh transporter (VAChT), modulating the active transport of ACh at the neuromuscular junction, and homozygous loss of VAChT leads to lethality. Methods: Exome sequencing (ES) was carried out to identify the molecular genetic cause of the disease in a 5-year-old male patient and histological, immunofluorescence as well as electron- and CARS-microscopic studies were performed to delineate the muscle pathology, which has so far only been studied in VAChT-deficient animal models. Results: ES unraveled compound heterozygous missense and nonsense variants (c.315G>A, p.Trp105* and c.1192G>C, p.Asp398His) in SLC18A3. Comparison with already-published cases suggests a more severe phenotype including impaired motor and cognitive development, possibly related to a more severe effect of the nonsense variant. Therapy with pyridostigmine was only partially effective while 3,4 diaminopyridine showed no effect. Microscopic investigation of the muscle biopsy revealed reduced fibre size and a significant accumulation of lipid droplets. Conclusions: We suggest that nonsense variants have a more detrimental impact on the clinical manifestation of SLC18A3-associated CMS. The impact of pathogenic SLC18A3 variants on muscle fibre integrity beyond the effect of denervation is suggested by the build-up of lipid aggregates. This in turn implicates the importance of proper VAChT-mediated synthesis and recycling of ACh for lipid homeostasis in muscle cells. This hypothesis is further supported by the pathological observations obtained in previously published VAChT-animal models.

Keywords: SLC18A1; congenital myasthenic syndrome; vesicular acetylcholine transporter (VAChT); lipid accumulation; muscle biopsy; CARS microscopy 


\section{Introduction}

Congenital myasthenic syndromes (CMS) are a heterogeneous group of rare genetic diseases characterized by impaired neuromuscular transmission. Clinical symptoms usually manifest in early childhood (most often within the first two years of life) and disease progression and severity varies greatly with ranges from severe "floppy infants" to the presence of minor symptoms such as ptosis and mild muscle weakness. On a general note, early onset might be associated with bulbar and respiratory symptoms, generalized fluctuating muscle hypotonia and ptosis with or without ophthalmoplegia. Undiagnosed, a proportion of neonatal onset cases develop infection-triggered critical situations with respiratory symptoms (apnoeas) resulting in hypoxic injury [1]. In adult patients, limb-girdle muscular weakness and fatigable weakness are prominent clinical features [2]. Presynaptic forms of CMS due to mutations in SLC5A7 and SLC18A3, impairing the synthesis and recycling of acetylcholine (ACh), have recently been described [3,4]. The SLC18A3 gene on chromosome 10q11.23 encodes the vesicular acetylcholine transporter (VAChT) modulating the active transport of ACh at the neuromuscular junction (NMJ). VAChT knockout in mice results in a lethal phenotype [5]. Interestingly, missense variants and whole gene deletions have been previously identified in CMS-patients, defining SLC18A3 as a CMS-causative gene (CMS subtype 21; MIM: \#617239), a molecular genetic observation in agreement with the above-mentioned function of the corresponding protein. These patients presented with features seen in presynaptic CMS forms, including ptosis, ophthalmoplegia, bulbar symptoms, fatigable weakness, decrement on low-frequency repetitive stimulation followed by a prolonged period of post-activation exhaustion, and apnoeic crises with moderate clinical improvement upon treatment with pyridostigmine (PS) [6,7]. Given that one patient additionally showed learning difficulties and left ventricular dysfunction [3], a phenotypical variability of pathogenic SLC18A3-mutations is likely. Along this line, a recent publication reported on a severe prenatal phenotype defined by akinesia, arthrogryposis, edema, and partial cleft palate based on a homozygous nonsense variant (c.1116C $>\mathrm{A}$; p.Cys372Ter) in the SLC18A3 gene [8]. Based on the genotype-phenotype correlation, the authors postulated that $S L C 18 A 3$ nonsense variants cause a more severe phenotype than missense variants. This assumption would be in line with previous studies showing a lethal phenotype in VAChT knockout mice [5].

Here, we present a long-term follow-up clinical description along with the first reported myopathological study of a paediatric patient presenting with compound heterozygous pathogenic variants (NM_003055:c.315G>A, p.Trp105* and c.1192G>C, p.Asp398His;) in SLC18A3. The benefit of PS is increasingly being reported for different CMS subtypes (depending on the underlying pathogenic variant) including ACh deficiency and glycosylation abnormalities, but only partial clinical improvement under PS treatment was observed in our patient.

\section{Materials and Methods}

Written informed consent for clinical description, inclusion of photographs of the patient in publications, genetic studies and use of the muscle biopsy for research purposes, was obtained from both patients' parents. This study was approved by the Ethics Committee of the University of Essen (19-9011-BO). The study was conducted in accordance with the principles of the Declaration of Helsinki 1975, as revised in 1983. The data that support the findings of this study are available from the corresponding author upon request.

A biopsy of the vastus lateralis muscle was performed for diagnostic purposes. Cryostat sections $(6 \mu \mathrm{m})$ were processed for routine histological and immunohistochemical staining and histochemical reactions including Oil Red O. Electron microscopy (EM) of ultrathin sections from glutaraldehyde-fixed, resin-embedded muscle was performed using a Philips CM10 transmission electron microscope [9]. 


\subsection{Molecular Genetic Studies and Review of the Literature}

A commercial gene panel analysis included 15 CMS causative genes: ALG14, ALG2, CHAT, CHRNA1, CHRNB1, CHRND, CHRNE, COLQ, DOK7, DPAGT1, GFPT1, GMPPB, MUSK, RAPSN, MYO9A.

For exome sequencing, genomic DNA extracted from peripheral blood and further captured using the Twist Human Core Exome Kit + RefSeq Panel (Twist Bioscience, San Francisco, CA) according to the manufacturer's protocol. Paired-end $(2 \times 150)$ sequencing was performed on a NextSeq550 sequencer (Illumina). Bioinformatic analysis, including alignment to the GRCh37/hg19 human reference genome, variant and copy number variant calling and variant filtering, was done using the Varvis software (Limbus Technologies $\mathrm{GmbH}$, Rostock). Targeted Sanger sequencing was subsequently performed for the index case and his parents to confirm biallelic variants in SLC18A3. Amplification of regions flanking the identified variants from genomic DNA was performed using two specific primer pairs (SLC18A3_Ex1_c315_F, 5'-CCCATAGTGCCCGACTACAT-3' and SLC18A3_Ex1_c315_R, 5' GCACGTCGTAGCTCATGC-3';SLC18A3_Ex1_c1192_F, 5'-CACACCTGCAGTGGCTGTA3', SLC18A3_Ex1_C1192_R-5'GCTCAAAGCCCAGCGAGT-3'). SLC18A3 variants are described on Refseq isoform NM_003055.3.

A review of the literature on $S L C 18 A 3$ patients was performed under the term "SLC18A3 and congenital myasthenic syndrome", revealing eight hits. After studying the articles, six relevant original articles and case reports were selected including all patients being reported to date $[3,8,10-12]$, as well as one article describing the NME pathology in $\mathrm{AChTKD}^{\mathrm{HOM}}$ mice [13]. No additional relevant articles were found in the citation lists of these articles.

\subsection{Coherent Anti-Stokes Raman Scattering (CARS) and SHG Microscopy and Immunofluorescence}

Six-micrometre-thick sections were used for the spectroscopic analysis. Sections were stored at $-80^{\circ} \mathrm{C}$ and under a constant nitrogen gas flow at room temperature dried for CARS and SHG measurements. No further sample preparation was applied for these studies.

The spectroscopic measurements were performed on a modified Leica TCS SP 8 CARS laser scanning microscope. A pulsed APE picoEmerald was used as laser source. We used the same measurement parameters as in our previous work [14]: briefly, for CARS measurements, the Stokes pulse was fix at $1064 \mathrm{~nm}$ and the pump pulse tunes to a wavelength between $804.0 \mathrm{~nm}$ to $826.4 \mathrm{~nm}$ with a step size of $0.7 \mathrm{~nm}$. Both lasers were fixed at a power of $900 \mathrm{~mW}$ at the output port. Further subsequent laser attenuation in the Leica system was to $25 \%$. Photomultipliertubes (PMTs) in backward and forward direction detected the generated CARS-signals in the wavelength range of $560-750 \mathrm{~nm}$. With a beam splitter $(560 \mathrm{~nm})$, the simultaneously generated SHG signal was separated and detected with PMTs in both directions in the wavelength range of $380-560 \mathrm{~nm}$. A $40 \times$ water immersion objective (IRAPO $40 \times / 1.10$ WATER) was used for CARS and SHG imaging. The measuring field was $292 \times 292 \mu \mathrm{m}(2048 \times 2048$ pixel $)$ with a pixel dwell time of approximately $10 \mu$ s. Before measuring spectra, single images were first taken with the pump pulse setting $811 / 814 / 817 \mathrm{~nm}$ (average of 3 images). This combination results in a CARS signal at $655 / 659 / 663 \mathrm{~nm}$ and corresponds to a wavenumber of $2932 / 2887 / 2841 \mathrm{~cm}^{-1}$. CARS spectra were acquired by tuning the pump laser from $804-826.4 \mathrm{~nm}\left(3039.3-2702.2 \mathrm{~cm}^{-1}\right)$ with a step size of $0.7 \mathrm{~nm}$ (averaging of two images).

\subsection{Statistical Evaluation of Muscle Fibre Calibers}

The Leica software LAS X (ver. 2.0) was used to manually measure the length and width of fully imaged muscle fibres from the CARS single images at $2932 \mathrm{~cm}^{-1}$. The muscle fibre calibre was averaged from the two lengths. For muscle fibres in longitudinal section, the width of the fibre was determined as the calibre. A total of 255 fibres were analysed for the SLC18A3-patient. As reference, 1457 fibres were analysed from five control samples. 


\subsection{Extraction of Spectra for the Determined Features}

The spectral CARS measurements were manually screened for conspicuous features. These were grouped according to their appearance (see results section) CARS spectra were extracted for the area of these features as well as for inconspicuous areas neighbouring these features, which were considered as normal reference. All spectra per area were normalized to one and subsequently averaged. A total of 283 spectra were used presented in the results section: A: $85, \mathrm{~B}: 80, \mathrm{C}: 24$, D: 54, reference: 40.

\subsection{Fluorescent Labelling of Cryo-Embedded Muscle Sections}

Ten-micrometre-thick sections of cryo-embedded muscle biopsies were fixed with $4 \%$ $\mathrm{PFA} / \mathrm{PBS}(\mathrm{pH}=7.4)$ for $15 \mathrm{~min}$ at room temperature (RT). Fixed sections were blocked with $1 \%$ BSA $/ 1 \%$ DMSO/PBS for $1 \mathrm{~h}$ at RT. HCS LipidTOX ${ }^{\mathrm{TM}}$ Green Neutral Lipid Stain (Invitrogen, Cat\# H34475, 1:50) for neutral lipid staining, mouse anti-human Spectrin antibody (1:100) for plasma membrane staining, and MitoTracker ${ }^{\mathrm{TM}}$ Deep Red FM (Invitrogen, Cat\# M22426, 1:50) for mitochondria staining were diluted in blocking buffer. As a second staining cocktail HCS LipidTOX ${ }^{\mathrm{TM}}$ Green Neutral Lipid Stain (Invitrogen, Cat\# H34475, 1:50) for neutral lipid staining, HCS LipidTOX ${ }^{\mathrm{TM}}$ Red Phospholipidosis Detection Reagent (Invitrogen, Cat\# H34351, 1:50) for phospholipid staining, and mouse anti-human Spectrin antibody (1:100) for plasma membrane staining were diluted in blocking buffer. The sections were incubated with the respective antibody cocktails for 4h at RT in the dark, followed by washing 3 times with blocking buffer for $15 \mathrm{~min}$ each. Sections stained with antibody cocktail 1 were stained over night at RT with AlexaFluor555 goat anti-mouse secondary antibody (Biolegend, Cat\# 405324, 1:200) while sections stained with antibody cocktail 2 were stained overnight at RT with AlexaFluor647 goat anti-mouse secondary antibody (Biolegend, Cat\# 405322, 1:200) in blocking buffer. All sections were washed 3 times with blocking buffer for 15 min each and stained with DAPI (Carl Roth, Cat\# 6335.1, $1 \mathrm{mg} / \mathrm{mL}, 1: 500$ ) in blocking buffer for $10 \mathrm{~min}$ at RT. Finally, all sections were washed 2 times with blocking buffer, and one time with water for $15 \mathrm{~min}$ each at RT and covered with fluorescence mounting medium (Agilent Technologies, Cat\# S302380-2).

\subsection{Confocal Laser Scanning Microscopy (CLSM) and Image Processing of Fluorescent-Labelled Muscle Sections}

For high-resolution microscopy of fluorescent-labelled muscle sections a Leica TCS SP8 confocal laser scanning microscope with acousto-optic tuneable filters, an acoustooptical beam splitter, internal hybrid detectors (HyD SP), and a LMT200 high precision scanning stage was used. Imaging of coverslip-embedded samples was performed via a Leica HC PL APO 63x/1.20 W CORR objective combined with digital zoom factors 1.0 or 2.0. Fluorescence signals were generated via sequential scans, exciting HCS LipidTOX ${ }^{\mathrm{TM}}$ Green Neutral Lipid Stain via an argon laser at $488 \mathrm{~nm}$ and detecting with an internal HyD at 500-550 nm. AlexaFluor555 counter-stained Spectrin or HCS LipidTOX ${ }^{\mathrm{TM}}$ Red Phospholipidosis Detection Reagent were excited by a diode-pumped solid-state laser at $561 \mathrm{~nm}$ and detected with internal HyDs at 600-650 nm. The third sequence for visualizing MitoTracker ${ }^{\mathrm{TM}}$ Deep Red FM or AlexaFluor647 counter-stained Spectrin involved a $633 \mathrm{~nm}$ helium-neon laser for excitation and internal $\mathrm{HyD}$ at 650-700 $\mathrm{nm}$ for detection. In the last sequential scan DAPI was excited via a $405 \mathrm{~nm}$ diode-pumped solid-state laser and detected by an internal HyD at 450-500 $\mathrm{nm}$. Generated images were deconvoluted with Huygens Professional (SVI) and 3D-reconstructed with Imaris software (Bitplane).

\section{Results}

\subsection{Clinical Findings}

The male patient presented in this study was born prematurely at $36+2$ weeks of gestation due to pathological cardiotocographical findings as the 2nd child of nonconsanguineous healthy parents (father is a Roma originating from Poland and the mother is Syrian-Aramaic from Turkey) after an uneventful pregnancy. His older brother is clini- 
cally unaffected. Intrauterine, club feet and fetal akinesia were already diagnosed. Due to respiratory insufficiency at birth (Apgar scores $1^{\prime} 4,5^{\prime} 4$ and 10' 6) the patient was initially mechanically ventilated over a period of 10 days. Birth measurements were within normal ranges. The patient additionally presented with arthrogryposis congenital (AMC), retrognathia, high arched palate, bilateral fluctuating ptosis, ophthalmoplegia, generalized muscular hypotonia, facial hypomimia, adducted thumbs and bulbar symptoms including feeding difficulties and weak cry. Due to respiratory insufficiency with recurrent apnoeas, cardiopulmonary reanimations with short periods of assisted ventilation were necessary during the first month post-partum. Echocardiography, electrocardiogram as well as electroencephalogram revealed normal results.

At the age of seven months, the clinical diagnosis of a CMS was established. The patient received a therapy with PS, which had a beneficial effect on ptosis and his muscle tone in extremities as well his facial hypomimia. His head control also improved, whereas bulbar and respiratory symptoms persisted. Therefore, tracheostoma was placed at the age of 11 months (Figure 1A). At the age of one year, PS therapy was paused because of uncertain efficiency and failed further motor development. However, given that his ptosis worsened, and muscular weakness decreased, PS therapy was restarted. Of note, an additional therapy with 3,4 diaminopyridine (DAP) and ephedrine was not effective. Motor development was profoundly impaired-he was able to turn himself from supine to prone position at the age of 23 months.

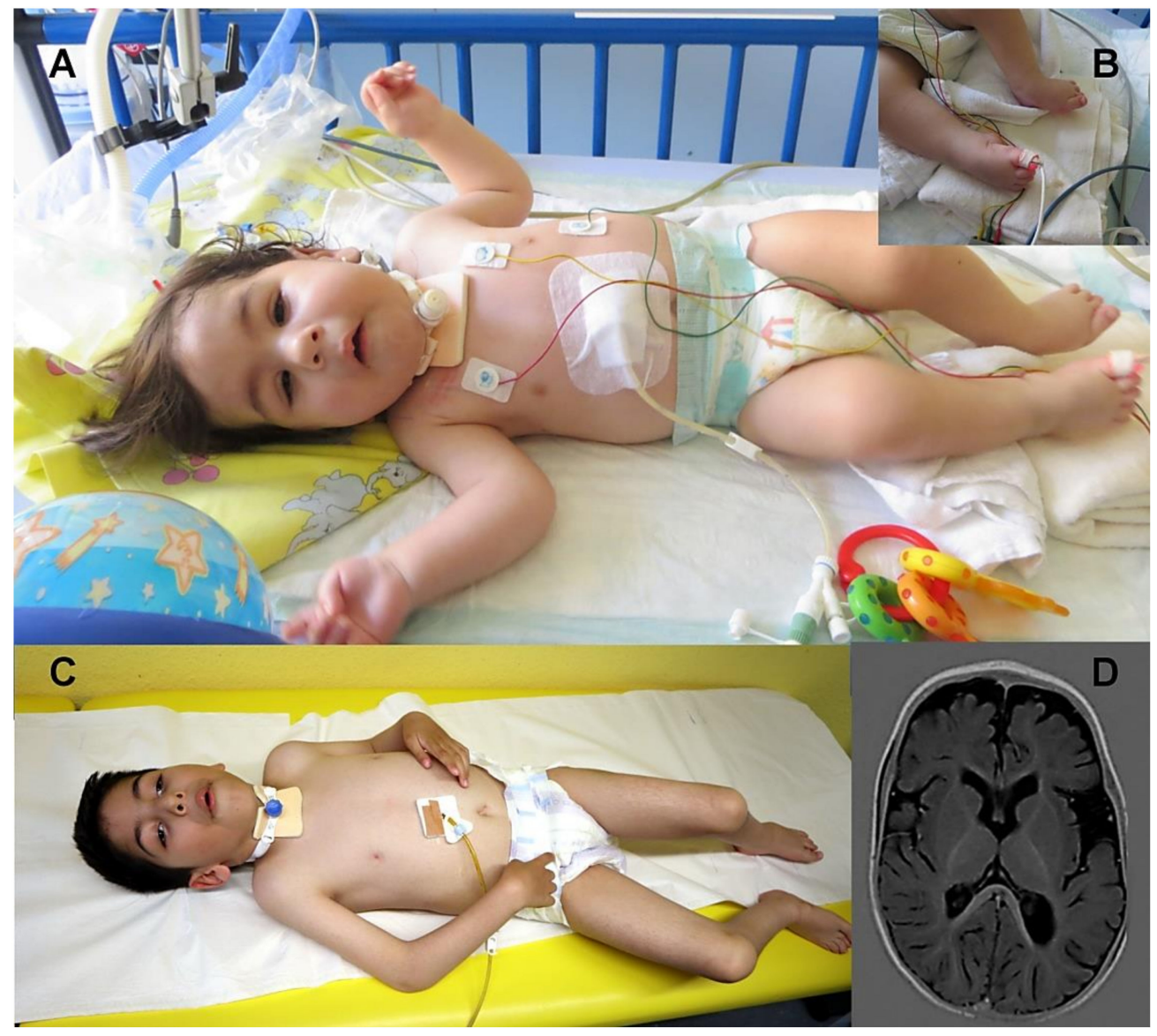

Figure 1. Clinical findings in the SLC18A3 patient. (A) Patient at the age of 11 months displaying facial features including high arched palate (not to be seen), hypomimia, both-sided ptosis and club feet (B). Feeding difficulties lead to percutaneous endoscopic gastrostomy (PEG)-tube feeding, and a tracheostoma was placed due to respiratory crises with apnoea. (C) At the age of 5 years, the facial hypomimia and ptosis persisted. Moreover, the patient developed joint contractures in the lower extremities (knee joints) and still needed tracheostoma. (D) Magnetic resonance imaging (MRI) performed at the age of 11 months revealed volume reduction with bitemporal accent. 
At last clinical follow-up at the age of 5 years 9 months, the boy still received PS therapy (dosage: $3 \mathrm{mg} / \mathrm{kg} /$ day) and was tracheostomized without assisted ventilation support. He had normal body measurements (weight on 25 th percentile, length on 42 nd percentile and head circumference on 33rd percentile) and was not able to sit independently but could turn himself from the prone to supine position and moved himself by rolling. His ptosis fluctuated during the day, muscular strength showed fluctuations from day to day, no diurnal fluctuations. His dysmorphic facial aspects (Figure 1 A,C) and joint contractures persisted. Due to ophthalmoplegia he could move his eyes only horizontally. His speech development appeared to be minimally impaired; he was able to pronounce single words at the age of 18 months. He visited a nursery with special medical support. A standardized intelligence testing was not performed but it was planned for him to visit a school with special support. His bulbar, muscular, and respiratory symptoms worsened during febrile or non-febrile infections. Cranial magnetic resonance imaging (MRI) showed mild generalised atrophy at the age of 11 months (Figure 1D). Repetitive stimulation at low frequency (3 Hz stimulus) showed decrement of $38 \%$ on Nervus medianus and $30 \%$ on Nervus tibialis. Because of only slight improvement under therapy with PS and due to a lack of effect of ephedrine and 3,4 DAP for the further diagnostic work-up (and to address the hypothesis of congenital myopathy), a muscle biopsy was performed at the age of three years.

\subsection{Molecular Genetics Findings}

Karyotyping and array-based comparative genomic hybridization (CGH) were initially performed and did not reveal any relevant chromosomal anomaly. Genetic testing of SMN1 was also normal. Gene panel analysis of 15 genes involved in CMS (see Materials and Methods section) did not reveal any pathogenic variant. An intronic variant in CHAT (c.699-108G > T) was identified but classified aslikely benign using ACMG criteria [15].

Exome sequencing in the index case revealed the presence of two heterozygous variants, c.315G>A (p.Trp105*) and c.1192G>C (p.Asp398His), in the single coding exon of SLC18A3. The nonsense variant (p.Trp105*) introduces an early premature termination codon at position 105, after the first transmembrane domain (Figure 2). The missense variant (p.Asp398His) replaces a highly conserved asparagine located in the 10th transmembrane domain of the VAChT by a histidine (Figure 2) and is one of the two pathogenic mutations originally reported by O'Grady et al. [3]. Sanger sequencing confirmed that both variants were each located on a different parental allele, with c.1192G>C, p.Asp398His inherited from the mother and c.315G >A, p.Trp $105^{*}$ inherited from the father.

\subsection{Review of the Patients Reported in the Literature}

Literature research revealed a current total of ten patients (including our patient) carrying pathogenic variants in SLC18A3 and published clinical information, which are summarized in Table 1 . In the cases of nonsense variants, both patients already died intrauterine and presented a combination of fetal akinesia and dysmorphic features (Patients 7 and 8; [8]). Two patients with homozygous missense variants had a severe neonatal presentation with feeding difficulties, respiratory problems and brain affection, and died within the first months of life or survived with severe motor and mental impairment (Patients 5 and 6; [11]). These siblings also harboured homozygous variants in NECAB2 (p.Arg307His), which were considered to not have an influence on neuromuscular function and were thus classified as irrelevant [11]. Five patients showed muscular hypotonia, ptosis, respiratory and feeding problems already neonatal (Patients 3, 4, 9 and 10) or during infancy (Patient 2) and improvement over time with good response to PS in all. Two patients responded to additional therapy with 3,4 DAP (Patients 3 and 9). These patients harboured whole gene deletions as compound heterozygous with missense variants (Patients 2 and 4 ) or homozygous missense variants (Patient 3). In the case of Patient 9 with compound heterozygous missense and nonsense variants, a good response to PS was reported (can walk with support). As highlighted in Table 1, in the case of our patient, compound heterozygosity of a nonsense and missense pathogenic variants was also detected. 
Notably, he showed a more severe phenotype, with only ability to sit for a few minutes at the age of five, and showing only partial response to PS, although this was already started at the age of 7 months.

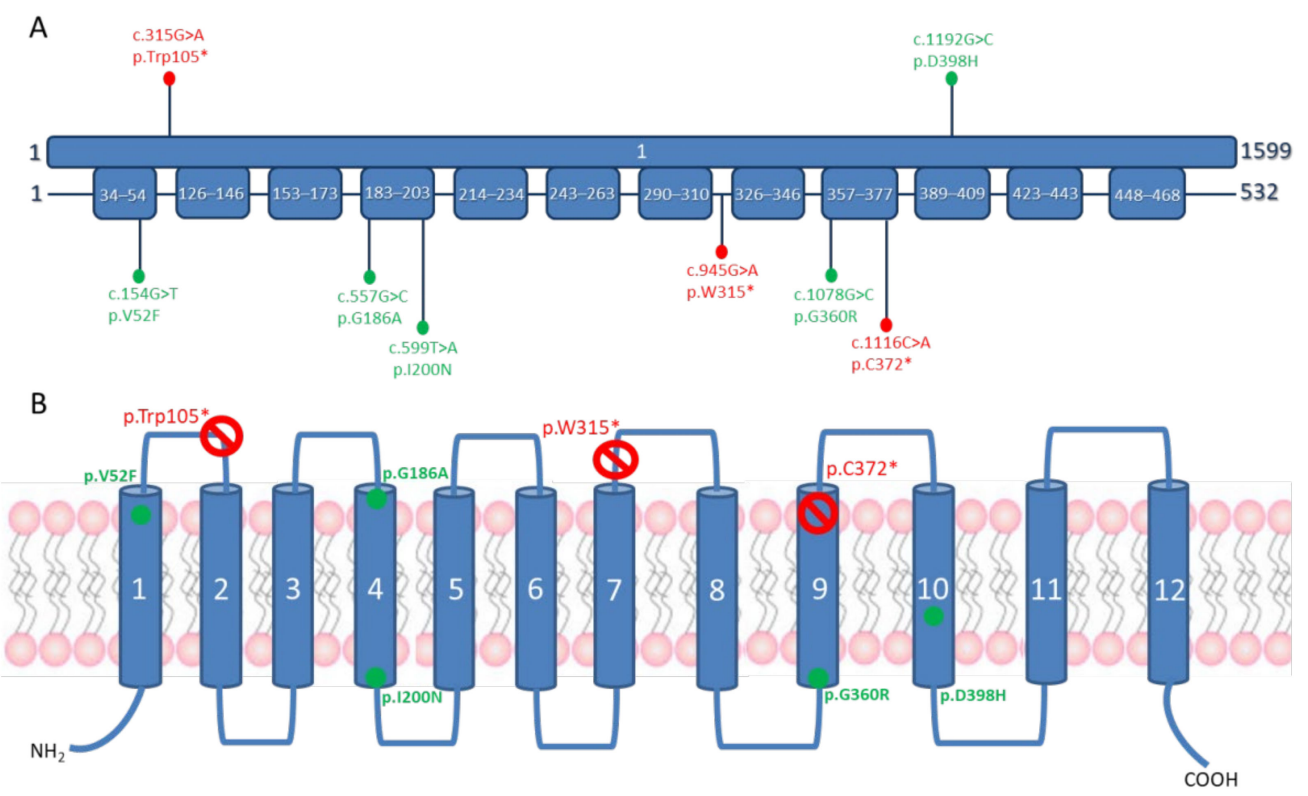

Figure 2. Overview of SLC18A3 pathogenic variants. (A) Schematic representation of SLC18A3 variants identified in this study (above) and reported in the literature (below) on the cDNA and protein. (B) Pathogenic variants represented on a schematic diagram of the SLC18A3 channel. Red: truncating variants; green: missense variants.

However, some specific phenotypic features are present in all cases: muscular hypotonia since birth, joint affection (arthrogryposis), ptosis, respiratory problems including apnoea and more severe disease course in homozygous mutations compared to compound heterozygous ones. Additionally, a brain involvement seems to be a part of the phenotype including delayed myelinisation and brain atrophy (Table 1). Fluctuation of symptoms during the day is not a prominent clinical feature, and in three patients additional nystagmus was present (Patient 3, 6 and 10).

\subsection{Light and Electron Microscopic Studies on Quadriceps Muscle Biopsy}

Histological studies by haematoxylin and eosin (H\&E) staining revealed variabilities in fibre size (Figure 3A). A profound accumulation of lipid droplets was identified by Oil Red O staining (Figure $3 \mathrm{~B}$ ). Investigation of semithin resin sections confirmed the considerable widening of the calibre spectrum combined with an accumulation of osmiophilic material in many muscle fibres (Figure 3C,D). 


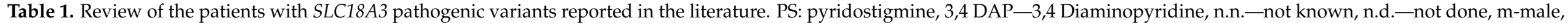
f-female,mo-motnhs, MRI—magnetic resonance imaging, CT—computer tomography.

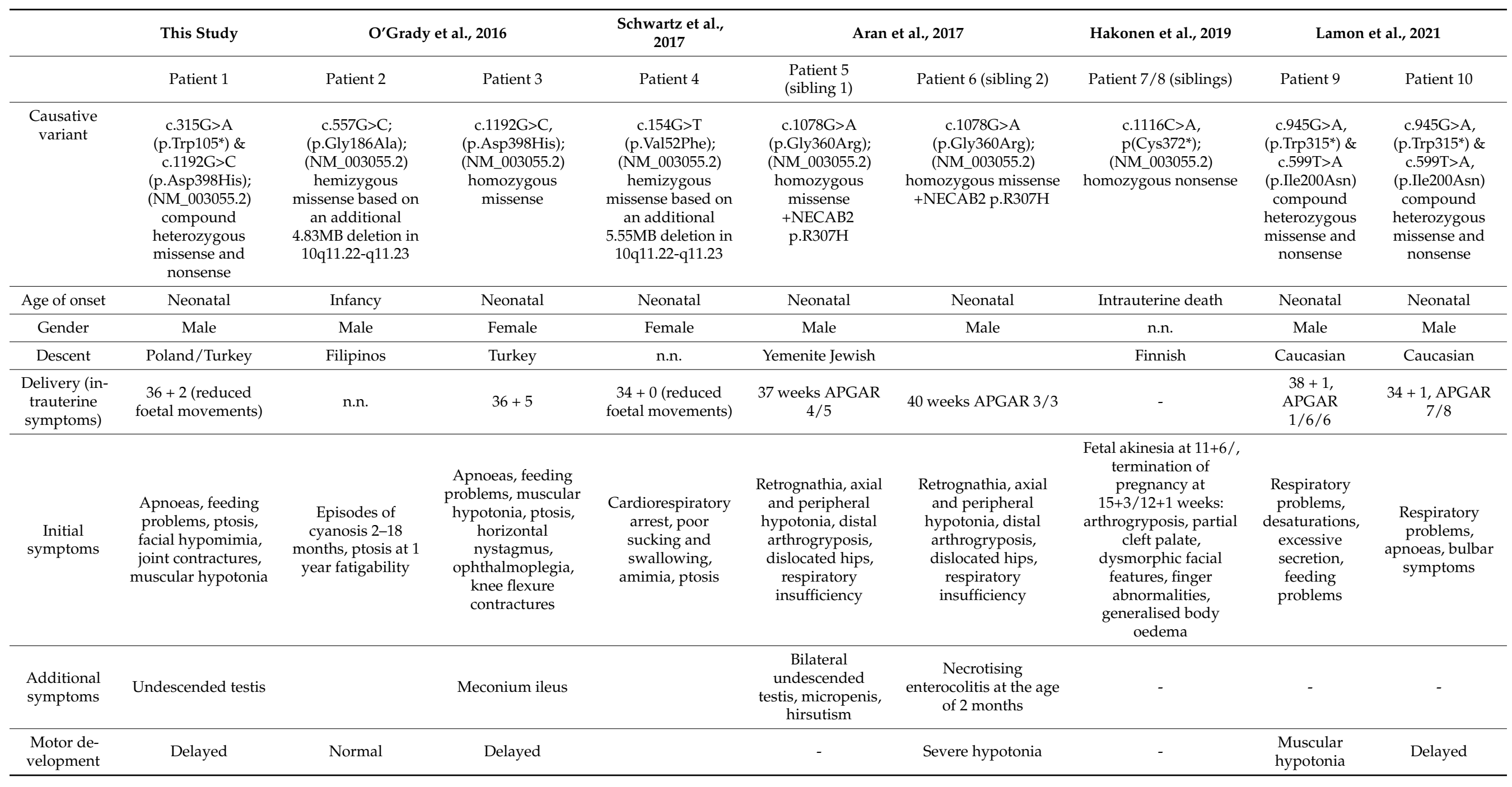


Table 1. Cont.

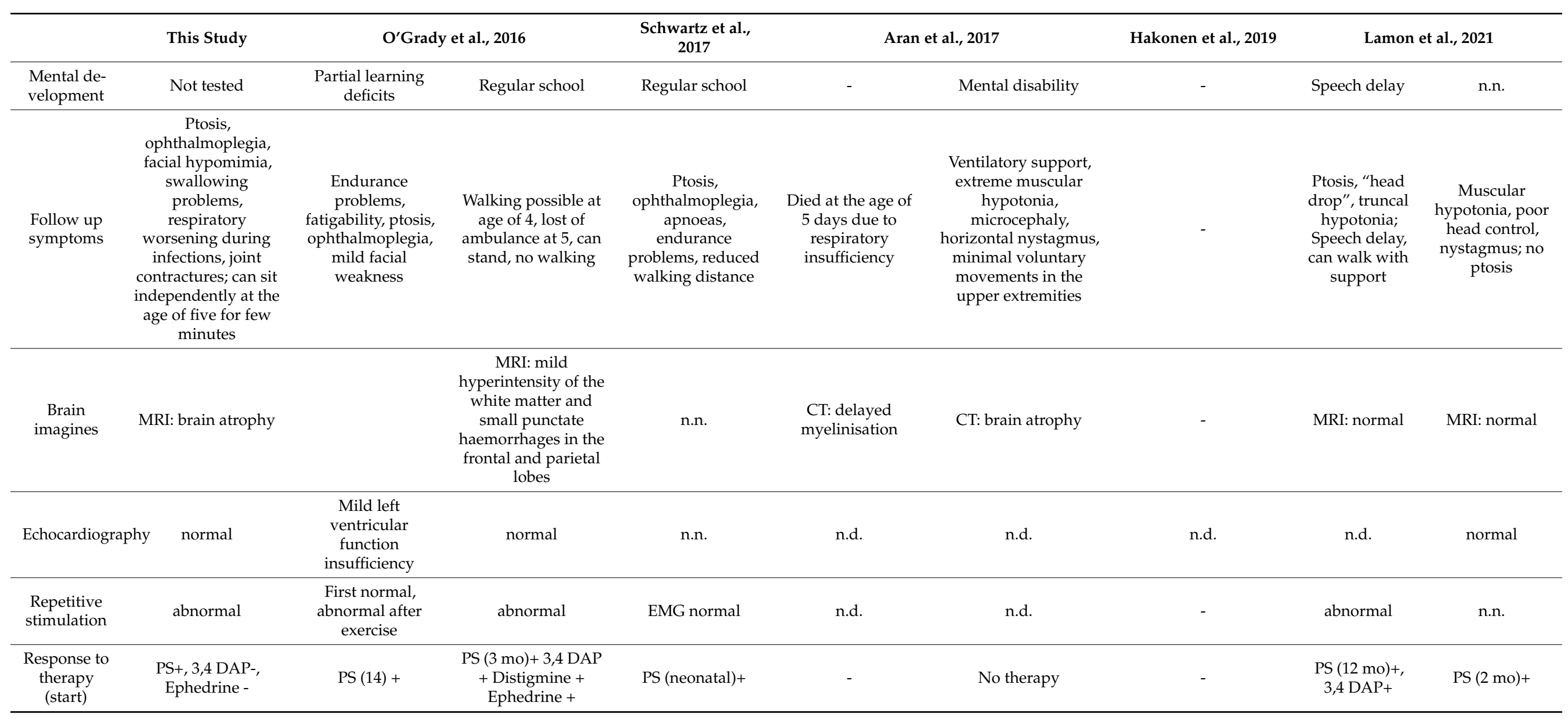



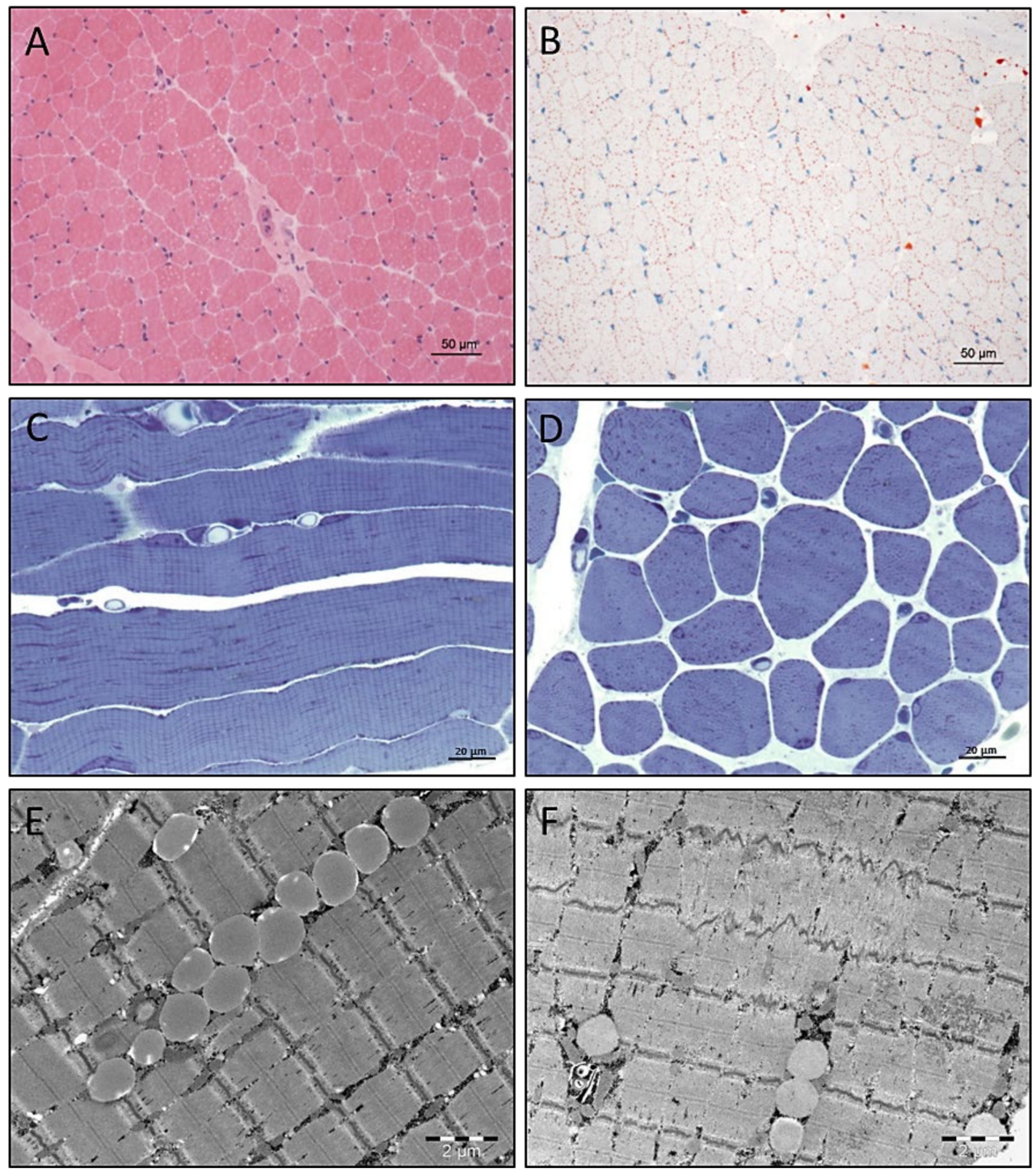

Figure 3. Results of histological and electron microscopic studies of quadriceps muscle derived from our SLC18A3 patient. (A) Widened spectrum of muscle fibre calibres identified by H\&E staining. (B) Increased number and size of lipid droplets in many muscle fibres identified by Oil Red O histochemistry. Scale bars $=50 \mu \mathrm{m}$. (C,D) Semithin resin section histology confirms the considerable widening of the calibre spectrum combined accumulation of osmiophilic material in many muscle fibres. Scale bars $=20 \mu \mathrm{m}$. Electron microscopic studies revealed $(\mathrm{E})$ increased number of intermyobrillar lipid droplets, often arranged in rows and (F) minor focal Z-band disintegration. Scale bars $=2 \mu \mathrm{m}$.

\subsection{Electron Microscopy}

Electron microscopic studies on the muscle biopsy derived from the SLC18A3 patient revealed the presence of intermyofibrillar lipid droplets (Figure $3 \mathrm{E}$ ) in addition to minor focal Z-band disintegration (Figure 3F).

\subsection{CARS Microscopic Studies on Quadriceps Muscle Biopsy}

We analysed the biochemical composition of muscle biopsies with coherent antiStokes Raman scattering (CARS), a non-linear variant of the Raman effect. This analysis does not require staining, markers or pre-treatment of the sample and is based on the excitation of molecular bonds associated with macromolecules, e.g., lipids or proteins. Second harmonic generation (SHG) is also a non-linear spectroscopic method that can be performed simultaneously with CARS. SHG signals indicate highly organized substances 
such as collagen [16]. In both methods, the signals are detected and displayed as contrast images at an excitation wavenumber.

Based on the CARS measurements, an average muscle fibre calibre of $36.5 \mu \mathrm{m} \pm 8.63 \mu \mathrm{m}$ was identified for the patient. However, the five controls showed an average fibre calibre of $43.92 \mu \mathrm{m} \pm 23.29 \mu \mathrm{m}$.

Within the patient biopsy, we detected four spectroscopically distinct features (Figure 4). Remarkably, three of these features represent changes in lipid distribution (Figure 4A-C). In most fibres, we observed regions of increased F-CARS signal intensity at $2847 \mathrm{~cm}^{-1}$ (Figure 4A) and assigned the wavenumber $2847 \mathrm{~cm}^{-1}$ to disordered lipids [17]. Likewise, we observed regions with altered lipid homeostasis between skeletal muscle fibres (Figure 4B) and within the extracellular matrix (Figure 4C) that also displayed an increased intensity at $2847 \mathrm{~cm}^{-1}$ (lipid). All lipid features also show low intensity around $2921 \mathrm{~cm}^{-1}$ (protein), a wavenumber characteristic for protein $[18,19]$. Of note, we also identified subsarcolemmal protein accumulation in the SLC18A3 patient-derived biopsy (Figure 4D), with signals within the range of $2921-2953 \mathrm{~cm}^{-1}$. For these protein accumulations, the intensity is lower by $2847 \mathrm{~cm}^{-1}$ (lipid) to the reference spectra.

A peak shift for lipids and proteins compared to the reference spectrum was identified. This peak shift can be simplified by the ratios of the intensities (Table 2). Thus, these features show a shift in the protein peak from $2921 \mathrm{~cm}^{-1}$ to higher wavenumbers $\left(2932-2953 \mathrm{~cm}^{-1}\right)$. The wavenumber around $2950 \mathrm{~cm}^{-1}$ is assigned to the asymmetric $\mathrm{CH} 3$ vibration. This vibration is counted among the possible vibration types of proteins [20-22]. We therefore assume a change in protein distribution, which is also reflected in the change in $\mathrm{CH} 3$ vibration. Moreover, in subsarcolemmal localized protein aggregates, protein intensities are increased compared to the reference, independent of the lipid signal. For the lipids, we also identified a shift from $2868 \mathrm{~cm}^{-1}$ to $2847 \mathrm{~cm}^{-1}$ in the feature spectra. For these features, the ratio of 2921 / 2868 is comparable to $2932 / 2968$ and 2921 / 2847 is comparable to $2932 / 2847$. This does not apply to the ratios of the reference. Thus, an increased signal at $2932 \mathrm{~cm}^{-1}$ occurs only in addition to the signal at $2921 \mathrm{~cm}^{-1}$.

Table 2. Ratio between intensities of wave numbers used for the detection of protein and lipid. The averaged spectra from Figure 4 were used to determine the ratios between wavenumbers characteristic of lipid and protein and the shifted wavenumbers.

\begin{tabular}{ccccccc}
\hline Ratios & $\mathbf{2 9 3 2 / 2 9 2 1}$ & $\mathbf{2 8 6 8 / 2 8 4 7}$ & $\mathbf{2 9 3 2 / 2 8 6 8}$ & $\mathbf{2 9 3 2 / 2 8 4 7}$ & $\mathbf{2 9 2 1 / 2 8 6 8}$ & $\mathbf{2 9 2 1 / 2 9 4 7}$ \\
\hline Inside fibres & 0.92 & 0.70 & 0.88 & 0.62 & 0.95 & 0.67 \\
Between fibres & 0.93 & 0.56 & 0.73 & 0.41 & 0.78 & 0.44 \\
$\begin{array}{c}\text { Inside connective } \\
\text { tissue }\end{array}$ & 1.01 & 0.57 & 0.86 & 0.49 & 0.85 & 0.49 \\
\hline $\begin{array}{c}\text { Subsarcolemmal } \\
\text { protein }\end{array}$ & 1.02 & 0.99 & 1.21 & 1.20 & 1.19 & 1.18 \\
\hline Reference & 0.86 & 1.14 & 0.88 & 1.00 & 1.02 & 1.16 \\
\hline
\end{tabular}

\subsection{Confocal Laser Scanning Microscopy (CLSM) and Image Processing of Fluorescent-Labelled} Muscle Sections

Staining of neutral lipids and the sarcolemma enabled the detection of lipid droplets lined up along the sarcolemma in the biopsy of our SLC18A3 patient. In addition, myofibre mitochondria showed lower neutral lipid contents than the sarcolemma (Figure 5). No sarcolemma-associated lipid droplets were observed in control muscle fibres. Notably, the SLC18A3 patient's sarcolemma-associated neutral lipid droplets are encapsulated in phospholipid membranes (but negative for Spectrin) (Figure 5). No sarcolemma-associated neutral lipid droplets encapsulated by phospholipid membranes were observed in healthy control samples. The finding of lipid droplets encapsulated by phospholipid membranes at the sarcolemmal area accords with the increased size of those compared to such within the sarcoplasm (two-tailed Mann-Whitney U-test $={ }^{* * * *} p<0.0001$ ) (Figure 6). Staining with $\alpha$-Bungarotoxin revealed only a few dot-like nicotinic ACh receptors at neuromuscular 
junctions in the SLC18A3 patient-derived biopsy compared to healthy control in which, in addition to the dot-like structures (open arrowheads), more extensive staining can also be observed (Figure 5C).

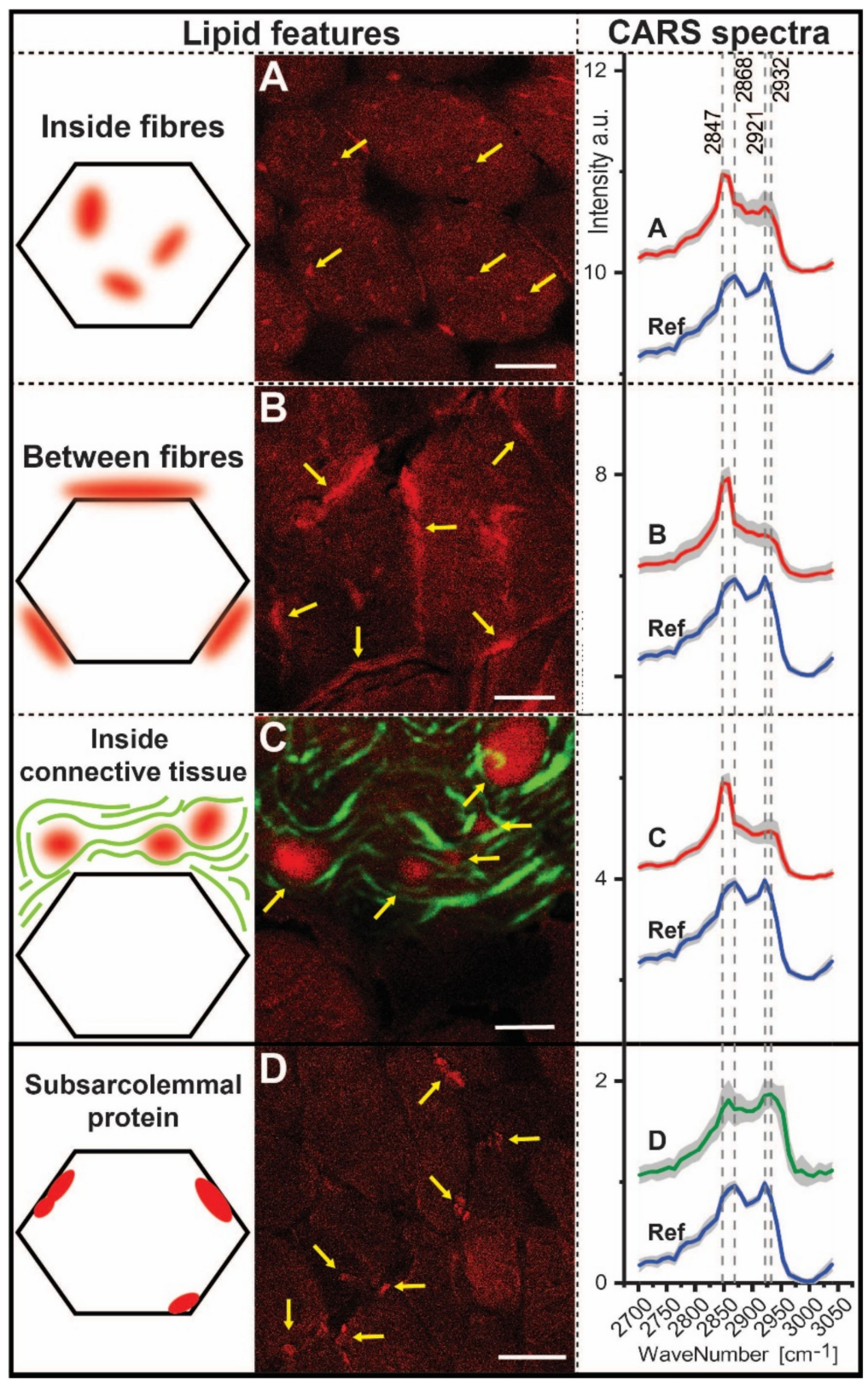

Figure 4. Spectroscopic findings in the muscle biopsies of the SLC18A3 patient determined from CARS and SHG measurements. CARS images in forward direction (F-CARS) are shown in red and F-SHG images in green. CARS/SHG images showing lipid (A-C) were taken at $2847 \mathrm{~cm}^{-1}$; otherwise $2953 \mathrm{~cm}^{-1}$ (D). (A) Lipid accumulation within a muscle fibre with high F-CARS signal at $2847 \mathrm{~cm}^{-1}$ (marked with arrows, Scale bar: $20 \mu \mathrm{m}$ ). (B) Lipid accumulation between muscle fibres with high F-CARS signal at $2847 \mathrm{~cm}^{-1}$ (marked with arrows, $10 \mu \mathrm{m}$ ). (C) Lipid accumulation within connective tissue with high F-CARS signal at $2847 \mathrm{~cm}^{-1}$ (marked with arrows). Collagen fibres are detected with F-SHG $(7.5 \mu \mathrm{m})$. (D) Subsarcolemmal protein accumulations with F-CARS signals at $2921 \mathrm{~cm}^{-1}$ and $2932 \mathrm{~cm}^{-1}(20 \mu \mathrm{m})$. A total of 283 CARS spectra were used; A: 85, B: 80, C: 24, D: 54, reference: 40. 

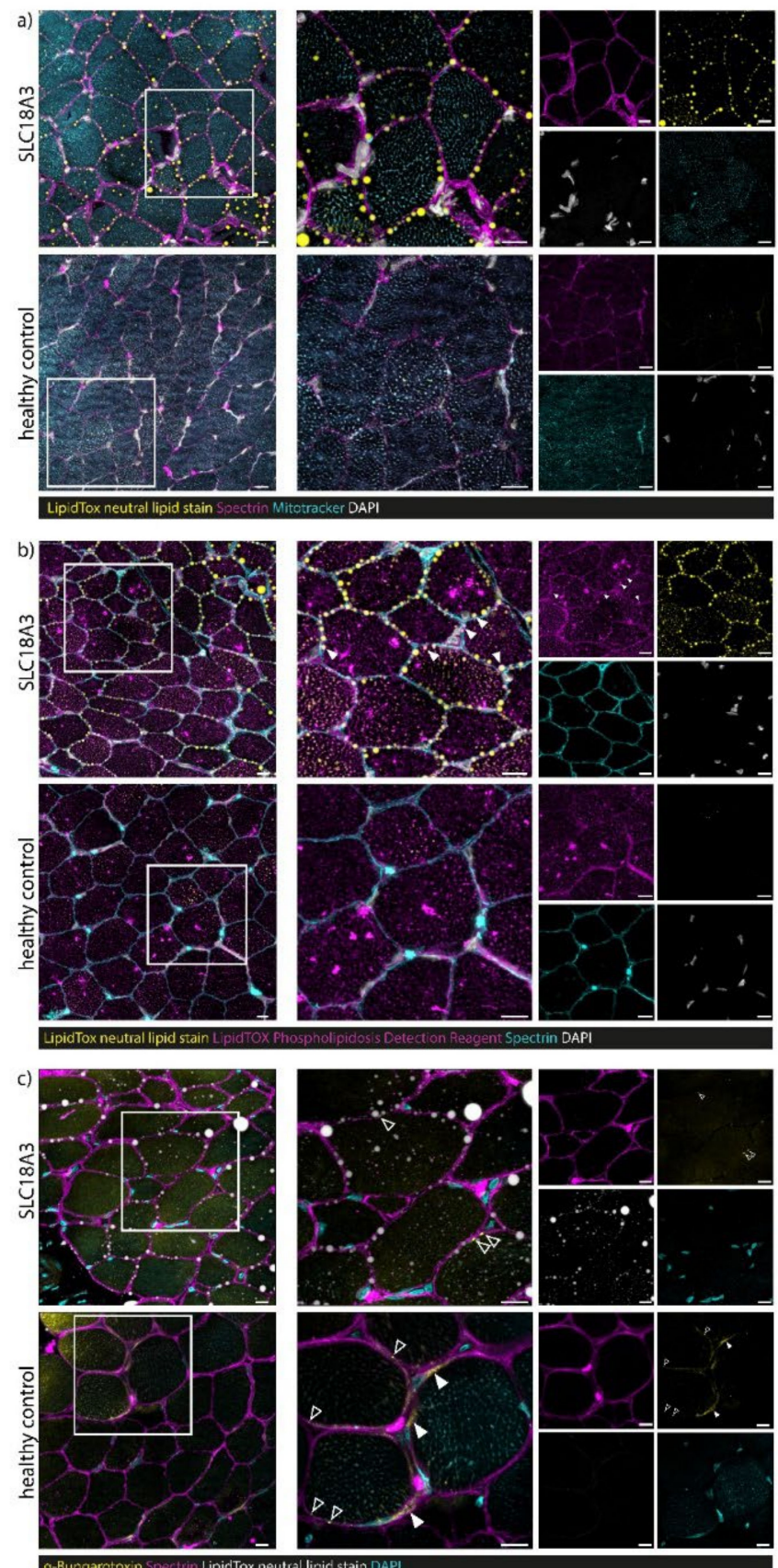

Figure 5. Immunofluorescence studies on muscle biopsy derived from the SLC18A3 patient. (a) Staining of neutral lipids (HCS LipidTox green neutral lipid stain, yellow) and muscle membrane (Spectrin, magenta) allows the detection of lipid droplets lined up along the sarcolemma in the biopsy of our SLC18A3 patient. Myofibre mitochondria (Mitotracker staining, turquoise) shows lower neutral lipid contents than the sarcolemma. No sarcolemma-associated lipid droplets were observed in healthy control samples. (b) The SLC18A3 patient's sarcolemma-associated neutral lipid droplets (HCS LipidTox green neutral lipid stain, yellow) are encapsulated in phospholipid membranes (HCS LipidTOX Red Phospholipidosis Detection Reagent, magenta, indicated via white arrowheads) but negative for spectrin (turquoise). No sarcolemma-associated neutral lipid droplets encapsulated by phospholipid membranes were observed in healthy control samples. (c) Staining with $\alpha$-Bungarotoxin (yellow) indicates only a few dot-like nicotinic acetylcholine receptors at neuromuscular junctions in SLC18A3 patient biopsy (open arrowheads) compared to healthy control in which, in addition to the dot-like structures (open arrowheads), more extensive staining can also be observed (filled arrowheads). Scale bars $=10 \mu \mathrm{m}$. 
a)

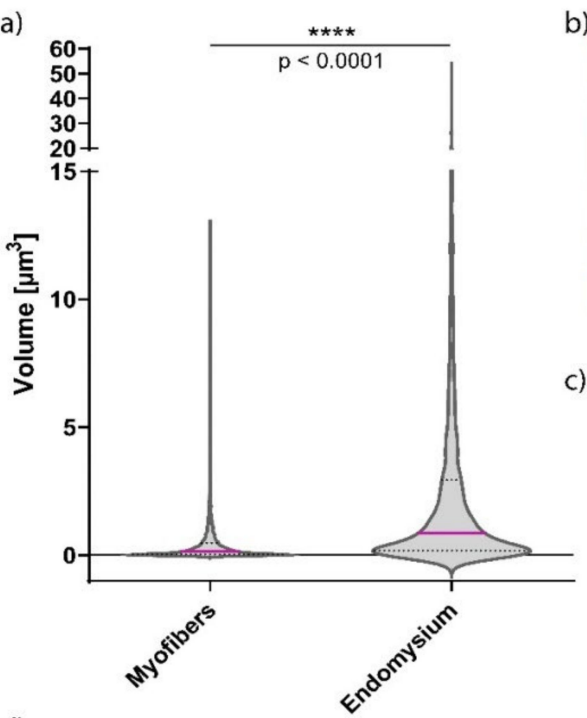

b)
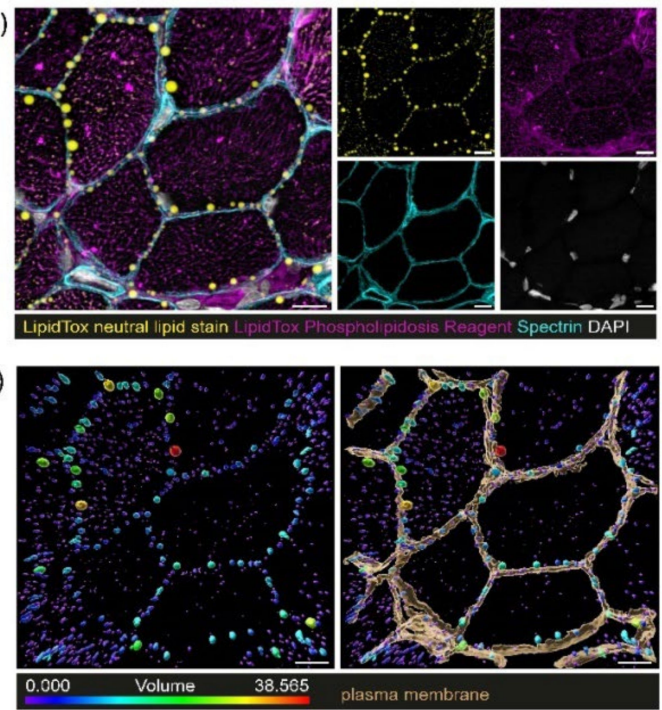

d)

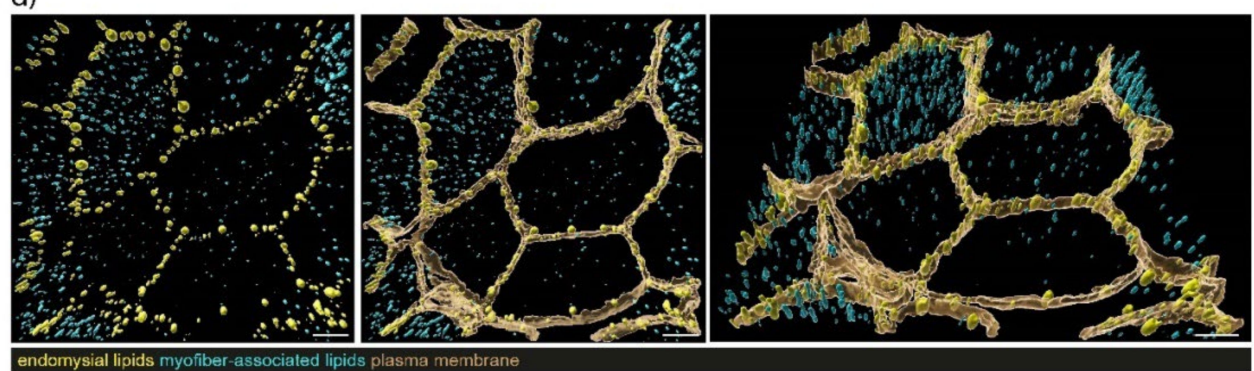

Figure 6. Volumetric size analysis of sarcolemma-associated lipid droplets and sarcoplasmic lipids. (a) Sarcolemma-associated lipid droplets have a significantly larger volume than lipid structures accumulating within the sarcoplasm ( $n=6$ individual field of views (FOW) from 3 independent technical replicates, $\mathrm{n}=4664$ myofiber associated lipid structures, $\mathrm{n}=1867$ sarcolemma-associated lipid droplets, two-tailed Mann-Whitney U-Test, ${ }^{* * * *} p<0.0001$. (b) Exemplary FOW for volumetric lipid structure analysis showing neutral lipids (HCS LipidTox green neutral lipid stain, yellow), phospholipids (HCS LipidTOX Red Phospholipidosis Detection Reagent, magenta), sarcolemma (Spectrin, turquoise), and nuclei (DAPI, white). (c) Optically isolated sarcolemma is shown in brown, while lipid structures are color coded according to their volume. (d) Sarcolemma-associated lipids (yellow) and sarcoplasmic lipids (turquoise) are identified and separated according to their distances to the plasma membrane (brown). Scale bars $=10 \mu \mathrm{m}$.

\section{Discussion}

\subsection{Phenotype Associated with Bi-Allelic SLC18A3 Variants}

The majority of SLC18A3 patients already showed onset of their symptoms neonatally, only one patient during infancy, with respiratory insufficiency, bulbar symptoms or apnoea as the dominating symptoms at disease onset. Of note, SLC18A3 patients tend to already have symptoms intrauterine and present with $A M C$, as was the case in our patient. Interestingly, fluctuation of the symptoms during the course of the day does not seem to be a prominent clinical feature, as is the case in most CMS mutations. Nystagmus was reported in three out of ten patients, but has not been described in other CMS cases to date. However, the SLC18A3-associated phenotype shares features of other known presynaptic mutations, such as CHAT and SLC5A7. In particular, the severe phenotype of SLC5A7 patients shares clinical similarities with $S L C 18 A 3$ patients including reduced intrauterine movements with AMC and only partial response to PS in some patients [4,23]. Wang and co-workers hypothesised that profound reductions in choline transporter activity in severely affected SLC5A7 infants and extremely low levels of mutant choline transporter 
at the NMJ would likely explain the extreme hypotonia, fatigability, lack of antigravity movements and respiratory insufficiency observed at birth [23]. Two SLC18A3 patients showed brain atrophy (Patients 1 and 6), both presenting apnoea neonatally as well. In our patient, no periods of prolonged hypoxia were documented although he suffered from repeated episodes of apnoea with the need for assisted ventilation. Moreover, the only patient with later onset and no respiratory insufficiency had mental impairment (Patient 2). Our patient did not undergo standardised, age-depended intelligence testing, but his speech development and cognitive function seems to be mildly impaired. Mental impairment and brain affection in presynaptic CMS with CHAT and SLC5A7 mutations seem to be based on multiple functions of $\mathrm{ACh}$, the choline transporter and VAChT, not just at the NMJ, but also in central nervous system [11,23-27]. Along this line, decreased VAChT expression may contribute not only to neurological symptoms, but also to cognitive alterations as in the case of mice with a 70\% reduction of VAChT expression [25].

The only-partial response to PS therapy could be explained by already reduced intrauterine movements causing structural muscular changes, in addition to a reduced amount of NMJs.

\subsection{Phenotype-Genotype Correlations}

In reported cases, including our patient, a genotype-phenotype correlation can be deduced: two patients with homozygous missense variants (Patients 5 and 6) presented with a severe phenotype but did not receive any therapy. Three patients with hemizygous or homozygous missense variants (Patients 2, 3, 4, 9 and 10) showed a delayed motor development, and four out of these five patients were able to walk under therapy with PS. In our patient, the combination of nonsense and missense variants in SLC18A3 led to a more severe phenotype compared to recessive missense variants only and with poorer response to therapy with PS in comparison to these patients. His nonsense variant caused an early premature termination codon (c.315G $>$ A; p.Trp $105^{*}$ ), presumably leading to nonsensemediated decay or expression of a very truncated form of the protein. However, Patients 9 and 10 also harboured compound heterozygous missense and nonsense variants. Given that the nonsense variant (c.945G >A, p.Trp315*) in these patients could lead to the expression of a less truncated protein with a residual function (compared to our patient), this might explain the manifestation of a less severe phenotype. Hakonen and co-workers postulated that this may lead to a milder phenotype in comparison to the nonsense variants, which might be caused by a residual function of the missense-mutant VAChT proteins [8]. However, systematic functional studies focussing on protein stability and activity are lacking to finally draw this conclusion. Nevertheless, the observation of intrauterine death in a SLC18A3 patient with a homozygous nonsense variant (c.1116C >A, p.Cys372*) accords with lethality of homozygous VAChT knock-out, whereas animals with a 70\% reduction of expression levels of VAChT are myasthenic and have cognitive deficits $[5,28]$. The latter aspect in turn suggests that minor levels of functional SLC18A3/VAChT proteins are sufficient to maintain survival associated with the manifestation of a paediatric neurological disease, thus in turn being in accordance with the hypothesis of Hakonen and co-workers [8].

Although there is a strong association between the nature of the genetic variant and the severity of the disease, one cannot exclude that other molecular factors (that have not yet been identified) might also impact the phenotypical presentation. Thus, further functional, biochemical and molecular genetic studies are crucial to systematically investigating the potential role of modifying factors (such as proteins or RNAs) that might moderate disease severity.

\subsection{Myopathology}

Interestingly, a primary vulnerability of the skeletal muscle based on histological and morphological findings was not reported for any of the nine patients reported thus far, and investigation of the muscle biopsy derived from our SLC18A3 patient revealed 
variabilities in fibre size on the histological and CARS-microscopic level, suggesting a vulnerability of muscle cells upon loss of expression of functional SLC18A3/VAChT protein. Indeed, skeletal muscle of $\mathrm{VAChT} \mathrm{Tel}^{\mathrm{del}}$ mutant mice showed a loss of normal myofibrillar architecture [5], a pathomorphological finding which was also observed in the biopsy of our patient on the electron microscopic level. Joviano-Santos and co-workers speculated that the affection of skeletal muscle fibres might be caused by a lack of proper ACh release in case of a mouse model with selective deletion of VAChT in motor neurons [29]. Moreover, CARS and electron microscopic studies revealed the presence of protein aggregates. This finding, along with changes of the cytoskeleton, might arise from denervation in terms of activated proteolysis in neurogenic atrophic muscle fibres [30].

Of note, our microscopic studies consistently revealed an irregular accumulation of lipid droplets between myofibrils, as well as adjacent to the sarcolemma. Thereby, droplets accumulating adjacent to the sarcolemma are larger. Further lipid staining experiments revealed that these neutral lipids are encapsulated by phospholipid membranes. Whereas the structural changes may be consequence of perturbed neuromuscular transmission as also patients harbouring SLC5A7 mutations present with myopathic changes or generalized muscular atrophy (in one patient electron microscopic studies showed targetoid fibres), no lipid droplets were evident even if patients presented with a severe phenotype with neonatal manifestation [23], as observed in our case. Muscle pathology based on denervation-reinnervation processes at the NMJs were evident in one patient with a milder phenotype, but also did not include lipid accumulation [4]. In our study, smaller NMJs in muscle fibres derived from the SLC18A3 patient were identified compared to such in muscle cells of a control biopsy specimen. Given that Freeman and co-workers could show that total ACh blockade in chicken embryos accompanied by reduced activation of the muscle had an adverse effect on muscle fibre differentiation and muscle fibres contained numerous large lipid droplets (similar as in our patient) [31], one might assume that proper VAChT-modulated acetylcholine transport into synaptic vesicles also has an impact on lipid homeostasis of muscle fibres (across species) during early development.

However, more functional and biochemical studies on SLC18A3 mutant or VAChTdepleted tissue would be necessary to unravel the exact underlying pathomechanism.

\section{Conclusions}

We report the phenotypical and myopathological findings in a $S L C 18 A 3$ patient presenting with compound heterozygous SLC18A3 missense and nonsense variants suffering from CMS subtype 21; comparison with already published cases revealed that the combination of missense and nonsense variants possibly leads to a more severe phenotype (also affecting treatment response) than recessive missense variants only. Microscopic studies revealed fibre size variations, considerable massive accumulation of neutral lipids of different sizes (sarcolemma-associated ones were larger and encapsulated by phospholipid membranes) in addition to minor Z-band disintegrations suggesting a myopathology. However, absence of SLC18A3 expression in skeletal muscle in combination with a detrimental effect of loss of VAChT on viability in human and mice might indicate the importance of the VAChT-mediated ACh transport into synaptic vesicles in turn impacting on NMJ-integrity and lipid-homeostasis in muscle fibres.

Author Contributions: Conceptualization, A.D.M. and A.R. and A.G. (Andrea Gangfuß); methodology, A.A., C.D., A.C. and J.W.; software, A.A. and A.G. (Anika Grüneboom); validation, A.G. (Anika Grüneboom), A.A. and E.F.; formal analysis, C.D.; investigation, C.D., J.W., A.G. (Anika Grüneboom), A.C., A.H. and A.R.; resources, N.K., E.F., J.W. and A.S.; clinical data curation, A.D.M., A.G. (Anika Grüneboom), H.K., U.S.-S., A.G. (Andrea Gangfuß), N.K.; writing-original draft preparation, A.D.M., C.D., J.W., A.G. (Anika Grüneboom), A.R.; writing-review and editing, all authors.; visualization. All authors have read and agreed to the published version of the manuscript.

Funding: This study was financed in the framework of the NME-GPS project by the European Regional Development Fund (ERDF). 
Institutional Review Board Statement: The study was conducted according to the guidelines of the Declaration of Helsinki, and approved by the Institutional Ethics Committee of University of Essen (19-9011-BO). The approval date was 30 April 2020.

Informed Consent Statement: Informed consent was obtained from subject involved in the study Written informed consent has been obtained from the patient's parents to publish this paper.

Data Availability Statement: The data that support the findings of this study are available from the corresponding author upon reasonable request.

Acknowledgments: We thank Eckhard Piegsa for referring the patient. We thank Swantje Hertel and Claudia Krude for their expert technical assistance. Moreover, we wish to thank the patient and his family for their willingness to participate in this study. Two authors of this publication are members of European Reference Network for Neuromuscular Diseases.

Conflicts of Interest: The authors declare no conflict of interest.

\section{References}

1. McMacken, G.; Whittaker, R.G.; Evangelista, T.; Abicht, A.; Dusl, M.; Lochmüller, H. Congenital myasthenic syndrome with episodic apnoea: Clinical, neurophysiological and genetic features in the long-term follow-up of 19 patients. J. Neurol. 2018, 265, 194-203. [CrossRef] [PubMed]

2. Kao, J.C.; Milone, M.; Selcen, D.; Shen, X.M.; Engel, A.G.; Liewluck, T. Congenital myasthenic syndromes in adult neurology clinic: A long road to diagnosis and therapy. Neurology 2018, 91, 1770-1777. [CrossRef] [PubMed]

3. O'Grady, G.L.; Verschuuren, C.; Yuen, M.; Webster, R.; Menezes, M.; Fock, J.M.; Pride, N.; Best, H.A.; Damm, T.B.; Turner, C.; et al. Variants in SLC18A3, vesicular acetylcholine transporter, cause congenital myasthenic syndrome. Neurology 2016, 87, $1442-1448$. [CrossRef] [PubMed]

4. Bauché, S.; O’Regan, S.; Azuma, Y.; Laffargue, F.; McMacken, G.; Sternberg, D.; Brochier, G.; Buon, C.; Bouzidi, N.; Topf, A.; et al. Impaired Presynaptic High-Affinity Choline Transporter Causes a Congenital Myasthenic Syndrome with Episodic Apnea. Am. J. Hum. Genet. 2016, 99, 753-761. [CrossRef]

5. De Castro, B.M.; De Jaeger, X.; Martins-Silva, C.; Lima, R.D.F.; Amaral, E.; Menezes, C.; Lima, P.; Neves, C.M.L.; Pires, R.G.; Gould, T.W.; et al. The Vesicular Acetylcholine Transporter Is Required for Neuromuscular Development and Function. Mol. Cell Biol. 2009, 29, 5238-5250. [CrossRef]

6. Vanhaesebrouck, A.E.; Beeson, D. The congenital myasthenic syndromes: Expanding genetic and phenotypic spectrums and refining treatment strategies. Curr. Opin. Neurol. 2019, 32, 696-703. [CrossRef]

7. Nicolau, S.; Milone, M. The Electrophysiology of Presynaptic Congenital Myasthenic Syndromes With and Without Facilitation: From Electrodiagnostic Findings to Molecular Mechanisms. Front. Neurol. 2019, 10, 257. [CrossRef]

8. Hakonen, A.H.; Polvi, A.; Saloranta, C.; Paetau, A.; Heikkilä, P.; Almusa, H.; Ellonen, P.; Jakkula, E.; Saarela, J.; Aittomäki, K. SLC18A3 variants lead to fetal akinesia deformation sequence early in pregnancy. Am. J. Med. Genet. Part A 2019, 179, ajmg. [CrossRef]

9. Mair, D.; Biskup, S.; Kress, W.; Abicht, A.; Brück, W.; Zechel, S.; Knop, K.C.; Koenig, F.B.; Tey, S.; Nikolin, S.; et al. Differential diagnosis of vacuolar myopathies in the NGS era. Brain Pathol. 2020, 30, 877-896. [CrossRef]

10. Schwartz, M.; Sternberg, D.; Whalen, S.; Afenjar, A.; Isapof, A.; Chabrol, B.; Portnoï, M.-F.; Heide, S.; Keren, B.; Chantot-Bastaraud, S.; et al. How chromosomal deletions can unmask recessive mutations? Deletions in $10 \mathrm{q} 11.2$ associated with CHAT or SLC18A3 mutations lead to congenital myasthenic syndrome. Am. J. Med. Genet. Part A 2018, 176, 151-155. [CrossRef]

11. Aran, A.; Segel, R.; Kaneshige, K.; Gulsuner, S.; Renbaum, P.; Oliphant, S.; Meirson, T.; Weinberg-Shukron, A.; Hershkovitz, Y.; Zeligson, S.; et al. Vesicular acetylcholine transporter defect underlies devastating congenital myasthenia syndrome. Neurology 2017, 88, 1021-1028. [CrossRef]

12. Lamond, A.; Buckley, D.; O'Dea, J.; Turner, L. Variants of SLC18A3 leading to congenital myasthenic syndrome in two children with varying presentations. BMJ Case Rep. 2021, 14, e237799. [CrossRef]

13. Leite Schetino, L.P.; de Castro Fonseca, M.; Magalhães Gomes, M.P.S.; Costa Valadao, P.A.; de Camargo, W.L.; Rodrigues, H.A.; Andrade, J.N.; Arantes-Costa, F.M.; Naves, L.A.; Prado, C.M.; et al. Evaluation of the neuromuscular junction in a middle-aged mouse model of congenital myasthenic syndrome. Muscle Nerve 2019, 60, 790-800. [CrossRef]

14. Hentschel, A.; Czech, A.; Münchberg, U.; Freier, E.; Schara-Schmidt, U.; Sickmann, A.; Reimann, J.; Roos, A. Protein signature of human skin fibroblasts allows the study of the molecular etiology of rare neurological diseases. Orphanet. J. Rare Dis. 2021, 16, 1-18. [CrossRef]

15. Richards, S.; Aziz, N.; Bale, S.; Bick, D.; Das, S.; Gastier-Foster, J.; Grody, W.W.; Hegde, M.; Lyon, E.; Spector, E.; et al. Standards and guidelines for the interpretation of sequence variants: A joint consensus recommendation of the American College of Medical Genetics and Genomics and the Association for Molecular Pathology. Genet. Med. 2015, 17, 405-424. [CrossRef] 
16. Ajeti, V.; Nadiarnykh, O.; Ponik, S.M.; Keely, P.J.; Eliceiri, K.W.; Campagnola, P.J. Structural changes in mixed Col I/Col V collagen gels probed by SHG microscopy: Implications for probing stromal alterations in human breast cancer. Biomed. Opt. Express 2011, 2, 2307. [CrossRef]

17. Rinia, H.A.; Burger, K.N.J.; Bonn, M.; Müller, M. Quantitative label-free imaging of lipid composition and packing of individual cellular lipid droplets using multiplex CARS microscopy. Biophys. J. 2008, 95, 4908-4914. [CrossRef]

18. Cheng, J.X.; Jia, Y.K.; Zheng, G.; Xie, X.S. Laser-scanning coherent anti-Stokes Raman scattering microscopy and applications to cell biology. Biophys. J. 2002, 83, 502-509. [CrossRef]

19. González Coraspe, J.A.; Weis, J.; Anderson, M.E.; Münchberg, U.; Lorenz, K.; Buchkremer, S.; Carr, S.; Zahedi, R.; Brauers, E.; Michels, H.; et al. Biochemical and pathological changes result from mutated Caveolin-3 in muscle. Skelet Muscle 2018, 8, 1-19. [CrossRef]

20. Saar, B.G.; Freudiger, C.W.; Reichman, J.; Stanley, C.M.; Holtom, G.R.; Xie, X.S. Video-rate molecular imaging in vivo with stimulated Raman scattering. Science 2010, 330, 1368-1370. [CrossRef]

21. Yu, Y.; Ramachandran, P.V.; Wang, M.C. Shedding new light on lipid functions with CARS and SRS microscopy. Biochim. Biophys. Acta Mol. Cell Biol. Lipids 2014, 1841, 1120-1129. [CrossRef]

22. Freudiger, C.W.; Yang, W.; Holtom, G.R.; Peyghambarian, N.; Xie, X.S.; Kieu, K.Q. Stimulated Raman scattering microscopy with a robust fibre laser source. Nat. Photonics 2014, 8, 153-159. [CrossRef]

23. Wang, H.; Salter, C.G.; Refai, O.; Hardy, H.; Barwick, K.E.S.; Akpulat, U.; Kvarnung, M.; Chioza, B.A.; Harlalka, G.; Taylan, F.; et al. Choline transporter mutations in severe congenital myasthenic syndrome disrupt transporter localization. Brain 2017, 140, 2838-2850. [CrossRef]

24. Schara, U.; Christen, H.-J.; Durmus, H.; Hietala, M.; Krabetz, K.; Rodolico, C.; Schreiber, G.; Topaloglu, H.; Talim, B.; Voss, W.; et al. Long-term follow-up in patients with congenital myasthenic syndrome due to CHAT mutations. Eur. J. Paediatr. Neurol. 2010, 14, 326-333. [CrossRef]

25. De Castro, B.M.; Pereira, G.S.; Magalhães, V.; Rossato, J.I.; De Jaeger, X.; Martins-Silva, C.; Leles, B.; Lima, P.; Gomez, M.V.; Gainetdinov, R.; et al. Reduced expression of the vesicular acetylcholine transporter causes learning deficits in mice. Genes Brain Behav. 2009, 8, 23-35. [CrossRef]

26. Pascual, J.M.; Liu, P.; Mao, D.; Kelly, D.I.; Hernandez, A.; Sheng, M.; Good, L.B.; Ma, Q.; Marin-Valencia, I.; Zhang, X.; et al. Triheptanoin for glucose transporter type I deficiency (G1D): Modulation of human ictogenesis, cerebral metabolic rate, and cognitive indices by a food supplement. JAMA Neurol. 2014, 71, 1255-1265. [CrossRef]

27. Liu, X.; Shi, Y.; Niu, B.; Shi, Z.; Li, J.; Ma, Z.; Wang, J.; Gong, P.; Zheng, A.; Zhang, F.; et al. Polymorphic variation in CHAT gene modulates general cognitive ability: An association study with random student cohort. Neurosci. Lett. 2016, 617, 122-126. [CrossRef]

28. Prado, V.F.; Martins-Silva, C.; de Castro, B.M.; Lima, R.F.; Barros, D.M.; Amaral, E.; Ramsey, A.; Sotnikova, T.D.; Ramirez, M.R.; Kim, H.-G.; et al. Mice Deficient for the Vesicular Acetylcholine Transporter Are Myasthenic and Have Deficits in Object and Social Recognition. Neuron 2006, 51, 601-612. [CrossRef]

29. Joviano-Santos, J.V.; Kljakic, O.; Magalhães-Gomes, M.P.S.; Valadão, P.A.C.; de Oliveira, L.R.; Prado, M.A.M.; Prado, V.F.; Guatimosim, C. Motoneuron-specific loss of VAChT mimics neuromuscular defects seen in congenital myasthenic syndrome. FEBS J. 2021, 288, 5331-5349. [CrossRef]

30. Jesse, C.M.; Bushuven, E.; Tripathi, P.; Chandrasekar, A.; Simon, C.M.; Drepper, C.; Yamoah, A.; Dreser, A.; Katona, I.; Johann, S.; et al. ALS-Associated Endoplasmic Reticulum Proteins in Denervated Skeletal Muscle: Implications for Motor Neuron Disease Pathology. Brain Pathol. 2017, 27, 781-794. [CrossRef]

31. Freeman, S.S.; Engel, A.G.; Drachman, D.B. Experimental acetylcholine blockade of the neuromuscular junction. Effects on end plate and muscle fiber ultrastructure. Ann. N. Y. Acad. Sci. 1976, 274, 46-59. [CrossRef] [PubMed] 